\title{
Exosomes Derived from Dental Pulp Stem Cells Accelerate Cutaneous wound Healing by Enhancing Angiogenesis via Cdc42/p38 MAPK Pathway
}

\section{Ziyu Zhou}

Sun Yat-sen University https://orcid.org/0000-0003-3971-2665

Jianmao Zheng

Sun Yat-Sen University

Danle Lin

Sun Yat-Sen University

Yanan Chen

Sun Yat-Sen University

Xiaoli Hu ( $\square$ huxiaol3@mail.sysu.edu.cn )

Sun Yat-sen University https://orcid.org/0000-0002-0462-7500

\section{Research}

Keywords: Exosomes, Wound healing, Angiogenesis, p38 MAPK pathway, DPSCs

Posted Date: October 29th, 2021

DOl: https://doi.org/10.21203/rs.3.rs-985814/v1

License: (c) (i) This work is licensed under a Creative Commons Attribution 4.0 International License. Read Full License 


\section{Abstract}

Background: Skin wound healing is a common challenging clinical problem and need advanced treatment strategies. Here, we investigated the therapeutic effects of exosomes derived from dental pulp stem cells (DPSC-Exos) on cutaneous wound healing and the underlying mechanisms.

Methods: The effects of DPSC-Exos on cutaneous wound healing in mice were examined by measuring wound closure rates, histological and immunohistochemical analysis. A series of functional assays were performed to evaluate the effects of DPSC-Exos on the angiogenic activities of human umbilical vein endothelial cells (HUVECs) in vitro. TMT-based quantitative proteomic analysis of DPSCs and DPSC-Exos was performed. Gene ontology (GO) and KEGG pathway enrichment analysis were used to evaluate biological functions and pathways for the differentially expressed proteins in DPSC-Exos. Western blot was used to assess the protein levels of Cdc42 and p38 in DPSC-Exos-induced angiogenesis of HUVECs. SB203580, a p38 MAPK signaling pathway inhibitor, was employed to verify the role of p38 MAPK pathway in these processes.

Results: Histological and immunohistochemical staining revealed that DPSC-Exos accelerated wound healing by improving neovascularization. DPSC-Exos augmented the migration, proliferation, and capillary formation capacity of HUVECs. Proteomic data demonstrated that proteins contained in DPSCExos regulated vasculature development and angiogenesis. Pathway analysis showed that proteins expressed in DPSC-Exos were involved in several pathways including MAPK pathway. Western blotting demonstrated that DPSC-Exos increased the protein levels of Cdc42 and phosphorylation of p38 in HUVECs. SB203580 suppressed the angiogenesis of HUVECs induced by DPSC-Exos.

Conclusions: DPSC-Exos could accelerate cutaneous wound healing by enhancing the angiogenic properties of HUVECs via Cdc42/p38 MAPK signaling pathway.

\section{Introduction}

As the largest organ of the human body, skin provides protection, sensory and metabolic functions ${ }^{1}$. The injury of skin can cause both physical and mental damage in patient. An impaired skin wound healing process may lead to infection, prolonged wound healing, and scarring ${ }^{2}$. Classic stages of wound repair include inflammation, new tissue formation and remodeling ${ }^{3}$. During the proliferation phase, the formation of neoangiogenesis plays a crucial role $e^{4}$.

Exosomes from mesenchymal stem cells (MSCs) originated from bone marrow, adipose tissue and umbilical cord have been demonstrated to accelerate cutaneous wound healing by enhancing angiogenesis, re-epithelialization and collagen deposition ${ }^{5-7}$. Exosomes are nanovesicles of endocytic origin secreted by most cells in culture, with a diameter of 30-150 nm and known as messengers that transmit bioactive molecules such as nuclei acid and proteins to the recipient cells for cell-cell communication ${ }^{8}$. They contain abundant cargoes which may participate in regeneration through various 
mechanisms. From a material science perspective, these nanocarriers with a particular composition of functional molecules are natural carriers, featuring extraordinarily better biocompatibility and bioavailability than conventional manufactured materials ${ }^{9}$. The exosomal proteins such as Jagged 1 , Ang-2 and DMBT1 actively participated in therapeutic angiogenesis for tissue repair ${ }^{10-12}$. However, the use of stem cells is often limited by the source and harvesting of stem cells is highly invasive. It is useful to look for a new parent cell source from which it is easy to obtain abundant exosomes for tissue repair.

DPSCs show MSC-like behavior, including great potential for angiogenic effects ${ }^{13}$. It can be harvested from the extracted third molars noninvasively, which is an extremely accessible cell resource for development of a therapeutic approach. Indeed, accumulating evidence suggested that DPSCs exhibited great potential in central nervous system repair, stroke recovery, diabetes treatment, muscle regeneration and wound healing ${ }^{14-16}$. DPSC-Exos have been reported to show regenerative therapeutic potential for

applications in dental pulp tissue regeneration, neuroprotection and craniofacial bone healing ${ }^{17-19}$. Considering that exosomes are important mediators of cell activity, it would be meaningful to explore if DPSC-Exos have the capability to accelerate the healing process of cutaneous wound.

In this study, we determined the effects of DPSC-Exos on angiogenesis of HUVECs, which related to cutaneous wound healing. We employed TMT-labeled quantitative proteomic analysis to identify the protein expression profiles in DPSC-Exos compared with their parent cells. GO annotation and KEGG pathway enrichment analysis were used to evaluate biological functions and pathways for the differentially expressed proteins in DPSC-Exos. The aims of the study were to clarify 1) the role of DPSCExos on the cutaneous wound healing in vivo; 2) the effect of DPSC-Exos on the angiogenic activities of HUVECs in vitro; 3 ) the potential signaling pathway involved in DPSC-Exos-induced angiogenesis of HUVECS.

\section{Materials And Methods}

\section{Cell culture}

This study was approved by the Ethics Committee of Sun Yat-sen University. Human DPSCs were obtained from healthy pulp tissues harvested from wisdom teeth without caries, which were extracted from 10 donors, both male and female, 22-36 years old. Dental pulp tissues were minced and digested for isolation of DPSCs as previously reported ${ }^{20}$. DPSCs were cultured in a-MEM with $10 \%$ FBS (GIBCO, USA) and $1 \%$ penicillin-streptomycin (Sigma, USA). HUVECs were bought from ALLCELLS (Shanghai, China) and expanded in endothelial cell growth medium (EGM). For exosome co-cultures experiment, $10 \mu \mathrm{g} / \mathrm{ml}$ of exosomes were added to the culture medium of HUVECs. All cells were kept at $37^{\circ} \mathrm{C}$ in an incubator contained $5 \% \mathrm{CO}_{2}$.

\section{Exosomes isolation and identification}


DPSCs were washed with PBS and incubated in fresh serum-free a-MEM $48 \mathrm{~h}$ prior to isolation of exosome. The cell culture supernatant was then harvested and subjected to two centrifugation steps (2000 $\times$ rpm for $10 \mathrm{~min} ; 5000 \times$ rpm for $30 \mathrm{~min}$ ) to remove dead cells and cell debris. Exosome pellets were purified from the cleared supernatant by ultracentrifugation at $100,000 \times g$ for $1 \mathrm{~h}$ at $4^{\circ} \mathrm{C}$. An exosome pellet from about $12 \times 10^{6}$ cells was resuspended in $100 \mu \mathrm{L}$ cold PBS. Exosomes were either used immediately or stored at $-80^{\circ} \mathrm{C}$ until use. The exosome protein concentration was quantified by a BCA Protein Assay Kit (Bocai, Shanghai, China). The expression levels of exosomal markers CD9 and CD63 (Affinity Biosciences, USA) were measured by western blot analysis. Nanoparticle Tracking Analysis (NTA) was performed to determine the size distribution of exosomes.

\section{Transmission electron microscopy}

The morphology of exosomes was visualized by transmission electron microscopy (TEM). Exosomes suspended in PBS were placed onto formvar/carbon-coated nickel grids and incubated for $30 \mathrm{~min}$. The images of grids were acquired using an $\mathrm{H}-7650$ transmission electron microscope (HITACHI, Japan).

\section{Mouse skin wound model and treatment}

All animal experiments were performed in accordance with institutionally approved protocols for animal research (Animal Care and Use Committee of Sun Yat-sen University). Female C57BL/6 mice were purchased from the Guangdong Medical Laboratory Animal Center. Age-matched 8-week female mice from the same background were used in this study. After shaving, a $1 \mathrm{~cm} \times 1 \mathrm{~cm}$ full-thickness excisional skin wound was created with a pair of surgical scissors, on the back of the mice. After the surgery on day 1 , six mice were randomly divided into two groups and were subcutaneously injected with either DPSCExos (120 $\mu$ g per mouse) as treatment group or an equal volume of PBS (control group) around the wounds at 4 equally spaced injection sites.

\section{Wound healing evaluation}

To observe the wound-healing process, wounds were captured by a digital camera on days $0,3,5,7,9,12$ and 14 , wound-healing rates were calculated according to the following equation: wound-healing rate (\%) $=(A 0-A n) / A 0 \times 100$, where $A 0$ represented the area of initial wound area $(t=$ day 0$), A n$ represented the residual area of wound on a the certain day $(t=$ day $n)$. Rates of wound closure were quantified with ImageJ software.

\section{H\&E staining and immunohistochemical staining}

To examine the formation of new blood vessels, these mice were sacrificed at day 14 post-wounding. The skin specimens were harvested and photographed, then analyzed by H\&E staining and 
immunohistochemical staining. The tissues were fixed in 4\% paraformaldehyde solution, dehydrated with a series of graded ethanol and embedded in paraffin. Sections (10 $\mu \mathrm{m}$ thick) were stained with hematoxylin and eosin $(\mathrm{H} \& \mathrm{E})$ or angiogenesis-related protein CD31 to evaluate the vascularization. The slides were observed and photographed under an epifluorescence microscope (Zeiss, Germany). To compare the number of newly formed blood vessels from different groups, five random fields per section near wound edges were counted by using Image-Pro Plus 6 software.

\section{Exosomes uptake assay}

To visualize the endocytosis of exosomes by HUVECs, exosomes were stained with PKH26 (SigmaAldrich, St Louis, MO, USA) according to the manufacturer's instructions. Isolated exosomes were resuspended in $250 \mu \mathrm{L}$ diluent $\mathrm{C}, 1 \mu \mathrm{L}$ PKH26 was added and immediately mixed by gentle pipetting. The staining was stopped by addition of an equal volume of exosome-free FBS after incubation for 5 min at room temperature. The mixture was washed twice with PBS by ultracentrifugation $(100,000 \times g$ for $1 \mathrm{~h})$ and resuspended in $100 \mu \mathrm{L}$ exosome-free culture medium. The PKH26-labeled exosomes were then added to the HUVECs and incubated for $24 \mathrm{~h}$. After treatment, the cells were washed with PBS for three times and fixed with $4 \%$ paraformaldehyde for $10 \mathrm{~min}$, the cell nuclei were stained with 4, 6-diamidino-2phenylindole (DAPI) for $5 \mathrm{~min}$. The images of fluorescence-labeled exosome internalization were obtained by a confocal laser scanning microscope (Zeiss, Germany).

\section{Transwell migration assay}

For the transwell migration assay, transwell inserts with 8- $\mu$ m pore filters (Corning, USA) were used. HUVECs $\left(1 \times 10^{4}\right.$ cells/well) were seeded onto upper chambers containing $200 \mu \mathrm{L}$ medium supplemented with DPSC-Exos, SB203580, SB203580+ DPSC-Exos, or PBS. Concurrently, $500 \mu \mathrm{L}$ of cultured medium was added to the bottom chamber and incubated at $37^{\circ} \mathrm{C}$ for $24 \mathrm{~h}$. The non-migrated cells in upper chamber were gently removed with a cotton swab and the lower chamber was fixed with $4 \%$ paraformaldehyde for 10 min, stained with $0.1 \%$ crystal violet (MYM biological technology company limited, Beijing, China) for 30 min, washed with PBS twice, and imaged by an inversion microscope (Zeiss, Germany). The quantity of migrated cells was counted and analyzed using ImageJ software.

\section{Scratch wound assay}

HUVECs $\left(2 \times 10^{5}\right.$ cells/well) were plated on 12 -well plates and incubated at $37^{\circ} \mathrm{C}$ until $90 \%$ confluence. A micro-injury was scratched on the monolayer using a $200 \mu \mathrm{L}$ pipette tip and washed with PBS to remove the debris. Cells were treated with DPSC-Exos, SB203580, SB203580+ DPSC-Exos, or PBS prior to taking microscopic images at $0 \mathrm{~h}$. The level of migration was measured by the ratio of closure area to initial wound area $(t=0 h)$ as follows: migration area $(\%)=(A 0-A 6) / A 0 \times 100$, where $A 0$ represents the area of initial wound area, $A 6$ represents the residual area of wound at the metering point after six hours $(t=6 \mathrm{~h})$. 
The wound area was quantified using ImageJ software to calculate percentage wound closure and migration rate.

\section{Cell proliferation assay}

A Cell Counting Kit-8 (Dojindo, Japan) was used to assess cell proliferation. Concisely, HUVECs were seeded onto 96 -well plates $\left(1 \times 10^{3}\right.$ cells/well) in cultured medium treated with DPSC-Exos and SB203580. At day 1, 2, 3 and 4, the CCK-8 solution was added to HUVECs (10 $\mu \mathrm{L}$ per well) and cells were incubated at $37^{\circ} \mathrm{C}$ for $1 \mathrm{~h}$. The absorbance was measured at $450 \mathrm{~nm}$ by using a microplate reader (BioTek, USA) and the optical density values represented the proliferation level of HUVECs.

\section{Tube formation assay}

HUVECs $\left(1 \times 10^{4}\right.$ cells/well) were seeded onto $50 \mu$ L Matrigel (No.354277, Corning, USA) coated 96-well plate and treated with DPSC-Exos, SB203580, SB203580+ DPSC-Exos, or PBS $ه$ respectively. After 6 hours of culturing incubation, gels were observed to examine the image of tube formation. The total tube length and total loops were measured as the mean sum length and loops of capillary-like structures per well using ImageJ software.

\section{Protein extraction for proteomic analysis}

DPSCs seeded in $75 \mathrm{~cm}^{2}$ cell culture flasks were incubated in serum-free a-MEM for $48 \mathrm{~h}$ and then washed with PBS prior to use. DPSC-Exos were isolated from the culture medium of DPSCs respectively. Both DPSCs and DPSC-Exos samples contained three biological replicates. TMT-based quantitative proteomic analysis was conducted by Jingjie PTM BioLab (Hangzhou, China). All samples were sonicated on ice in lysis buffer ( $8 \mathrm{M}$ urea, $1 \%$ Protease Inhibitor Cocktail) for three times before centrifuged $(12000 \times g$ for 10 $\mathrm{min})$ at $4{ }^{\circ} \mathrm{C}$ to remove remaining debris. Subsequently, the supernatant was collected. A BCA protein assay was performed to determine the protein concentration.

\section{Trypsin digestion and TMT labeling}

The protein solution was reduced with $5 \mathrm{mM}$ dithiothreitol at $56^{\circ} \mathrm{C}$ for $30 \mathrm{~min}$, alkylated with $11 \mathrm{mM}$ iodoacetamide in the dark place at room temperature for $15 \mathrm{~min}$, and then diluted with $100 \mathrm{mM}$ TEAB until urea concentration $<2 \mathrm{M}$. Trypsin was added to the solution at 1:50 (w/w) trypsin-to-protein mass ratio and incubated overnight, then trypsin-to-protein mass ratio was changed to 1:100 (w/w). After trypsin digestion, the peptide was desalted, vacuum-dried, reconstituted in 0.5 M TEAB, and processed with a TMT kit. 


\section{HPLC fractionation and LC-MS/MS analysis}

Thermo Betasil C18 column ( $5 \mu \mathrm{m}$ particles, $10 \mathrm{~mm}$ ID, $250 \mathrm{~mm}$ length) was utilized to fractionate tryptic peptides into fractions by high $\mathrm{pH}$ reverse-phase HPLC. In short, peptides were initially separated with a gradient of $8 \%$ to $32 \%$ acetonitrile ( $\mathrm{pH} 9.0$ ) over 60 min into 60 fractions, and then combined into 6 fractions before dried by vacuum centrifugation.

For LC-MS/MS analysis, the tryptic peptides were dissolved in solvent A ( $0.1 \%$ formic acid) and separated by a reversed-phase analytical column. The gradient comprised an increase from $6 \%$ to $23 \%$ solvent $B$ ( $0.1 \%$ formic acid in $98 \%$ acetonitrile) over $26 \mathrm{~min}, 23 \%$ to $35 \%$ in $8 \mathrm{~min}$ and climbing to $80 \%$ in $3 \mathrm{~min}$ then holding at $80 \%$ for the last $3 \mathrm{~min}$. All processes were performed on an EASY-nLC 1000 UPLC system at a constant flow rate of $400 \mathrm{~nL} / \mathrm{min}$. The peptides were subjected to NSI source and analyzed by tandem mass spectrometry (MS/MS) using the UPLC system coupled online to Q ExactiveTM Plus (Thermo). Intact peptides were detected in the Orbitrap at 70,000 resolution and selected for MS/MS with the NCE setting as 28 , the fragments were then detected at a resolution of 17,500 . MS and MS/MS spectra were acquired in a data dependent manner. The automatic gain control (AGC) was set at $5 \times 10^{4}$. An electrospray voltage of $2.0 \mathrm{kV}$ was applied. The scan range was set from 350 to $1800 \mathrm{~m} / \mathrm{z}$ for full scan and the fixed first mass was set as $100 \mathrm{~m} / \mathrm{z}$.

\section{Database search and bioinformatics analysis}

The acquired MS/MS data were processed using Maxquant search engine (v.1.5.2.8). Tandem mass spectra were searched against human uniprot database concatenated with reverse decoy database. Trypsin/P was designated as cleavage enzyme; up to 4 missing cleavages were allowed. The mass tolerance for precursor ions was set to $20 \mathrm{ppm}$ (First search) and $5 \mathrm{ppm}$ (Main search), and the mass tolerance for fragment ions to $0.02 \mathrm{Da}$, respectively. False discovery rate (FDR) was adjusted to less than $1 \%$ and a minimum score of 40 was set for the identification of modified peptides.

Gene Ontology (GO) annotation proteome was carried out through the UniProt-GOA database (). Proteins were classified by GO annotation into three categories: biological process, cellular compartment and molecular function. InterProScan was used for functional annotation of identified proteins domain based on protein sequence alignment and the InterPro 0 domain database. For functional enrichment analysis, the Kyoto Encyclopedia of Genes and Genomes (KEGG, ) and Gene Ontology (GO, 0 were applied.

\section{Determination and inhibition of p38 MAPK signaling pathway}

A pharmacological p38 MAPK inhibitor, SB203580 (Selleck Chemicals), was used to assess the participation of the MAPK signaling pathway in the DPSC-Exos-induced effects on HUVECs. The compound was resuspended to $10 \mathrm{mM}$ in dimethyl sulfoxide (DMSO) and used at $10 \mu \mathrm{M}$. For control 
treatments $(0 \mu \mathrm{M}) 0.1 \%$ DMSO was used. For experiments assessing the expression of target proteins by Western blotting, HUVECs were plated in 6-well plates and pretreated with $10 \mu \mathrm{M}$ SB203580 for $6 \mathrm{~h}$. Since SB203580 inhibited p38 MAPK catalytic activity instead of phosphorylation of p38, the effect of SB203580 on p38 MAPK pathway was assessed by expression of hsp27 as a substrate ${ }^{21}$.

\section{Western blotting}

Total protein of cells was extracted with RIPA buffer (Bocai, Shanghai, China) and quantified with a BCA Protein Assay Kit (Bocai, Shanghai, China). The protein samples ( $5 \mu \mathrm{g}$ per lane) were electrophoresed with 4-20\% Bis-Tris sodium dodecyl sulfate polyacrylamide gel and transferred onto a $0.2 \mu \mathrm{m}$ PVDF membrane (Millipore). After blocking with $5 \%(\mathrm{w} / \mathrm{v})$ nonfat milk for $1 \mathrm{~h}$ at room temperature, the membranes were incubated with diluted primary antibodies overnight at $4{ }^{\circ} \mathrm{C}$. The primary antibodies and concentration used in this study are: Phospho-p38 (Cell Signaling, \#4511, 1:1000), p38 (Cell Signaling, \#8690, 1:1000), Phospho-HSP27 (Affinity, AF3080, 1:1000), HSP27 (Affinity, AF6082, 1:1000), Cdc42 (Affinity, DF6322, 1:1000), ß-Tubulin (Affinity, AF7011, 1:8000), FGD5 (Affinity, DF13013, 1:500), CD63 (Affinity, AF5117, 1:1000), CD9 (Affinity, AF5139, 1:1000), $\beta$-actin (Invitrogen, MA5-11869, 1:8000). Subsequently, the membrane was washed with TBST and treated with the appropriate secondary antibody (Asbio) for $1 \mathrm{~h}$ at room temperature. Bands on the blots were visualized using GeneGnome XRQ (Syngene, UK). The ImageJ software (National Institutes of Health, USA) was utilized to quantify the density of the bands.

\section{Statistical analysis}

All experiments were performed with at least three replicates per group and each in vitro experiment was repeated three times. All values were expressed as the mean \pm standard deviation (SD). Two groups were evaluated by Student's $t$-tests and more than two groups were compared by one-way ANOVAs using SPSS 25.0 (SPSS Inc., USA). $p<0.05$ was considered statistically significant.

\section{Results}

\section{Identification of DPSC-Exos}

Exosomes derived from DPSCs were characterized by TEM, western blotting and NTA. TEM images revealed that vesicles isolated from culture supernatant of DPSCs exhibited a spherical morphology (Figure 1A). Western blotting analysis demonstrated that these vesicles were positive for exosomal surface markers CD9 and CD63 (Figure 1B). The result of NTA showed that the diameters of these nanoparticles ranged from 50-100nm (Figure 1C). Taken together, these results confirmed their identity as exosomes. 


\section{DPSC-Exos promoted cutaneous wound healing in mice by promoting angiogenesis}

We investigated the effects of DPSC-derived exosomes on the cutaneous wound healing by using a fullthickness wound model on mice. A significantly faster wound closure rate was observed when DPSCExos were injected (Figure 2A, B). Skin images from the underside of the wounds which was collected 14 days post-wounding demonstrated that those DPSC-Exos-treated showed much more newly formed blood vessels comparing to the control group (Figure 2C). Furthermore, we performed H\&E staining and immunohistochemistry staining at the wound site. A considerable amount of capillary-structure blood vessels (Figure 2D, E) and a higher expression of CD31 in DPSC-Exos-treated group (Figure 2F, G) confirmed that DPSC-Exos enhanced local angiogenesis. These findings suggested that DPSC-derived exosomes could accelerate in vivo wound healing by promoting angiogenesis.

\section{DPSC-Exos enhanced the angiogenic activities of HUVECs}

To explore the effect of DPSC-Exos on HUVECs behavior in vitro, we first monitored the internalization of exosomes into these cells. After 24h incubation with PKH26-labeled DPSC-Exos, fluorescence microscopy analysis revealed red fluorescent spots in the cytoplasm around the nucleus of HUVECs, which indicated that HUVECs were able to take up DPSC-Exos (Figure 3A). For functional study, we next assessed how DPSC-Exos modulated HUVEC angiogenesis including migration, proliferation, and tube formation. Transwell migration assay (Figure 3B, C) and scratch wound assay revealed that DPSC-Exos significantly up-regulated the cell motility of HUVECs (Figure 3D, E). CCK-8 assay was utilized to examine the proliferation of cells within 4 days of culture time, which showed that DPSC-Exos increased the proliferative capacity of HUVECs (Figure 3F). DPSC-Exos treatment also improved tube formation in HUVECs as the total tube length and total loops increased in the DPSC-Exo-treated group (Figure 3G, H, I). All these results indicated that DPSC-Exos enhanced the migration, proliferation and tube formation ability of HUVECs.

\section{Proteomic analysis of DPSCs and DPSC-Exos}

Since the mechanism of DPSC-Exos enhancing angiogenesis still remains unknown, we employed TMTlabeled quantitative proteomic analysis to identify the protein expression profiles in DPSC-Exos compared with their parent cells. A total of 4592 proteins were quantified, including 1159 up-regulated and 460 down-regulated (Figure 4A). In addition, a volcano plot showing statistically sizable difference of expressed proteins between DPSC-Exos and DPSCs was conducted (Figure 4B). The change in differential expression level exceeding 1.5 was regarded as the change threshold for significance.

To thoroughly determine the proteins we gained in this data, Gene Ontology (GO) analysis and KEGG pathway analysis was performed to classify the biological functions and characteristics of them and 
their molecular interaction networks. The consequences of GO classification revealed that proteins involved in various biological processes in relation to wound healing such as positive regulation of cell motility, migration, proliferation, vasculature development and angiogenesis, were remarkably increased in DPSC-Exos (Figure 4C). KEGG pathway analysis showed that proteins contained in DPSC-Exos were invoved in several pathways includig MAPK signaling pathway (Figure 4D and Additional file 1). We treated HUVECs with DPSC-Exos and found a significant increase in expression of Cdc42 and phosphorylated-p38 (Figure 4E, F). Cdc42 is required for the activation of p38 and plays a key role on cell migration and proliferation ${ }^{22,23}$. Taken together these findings prompted our hypothesis that $\mathrm{Cdc} 42 / \mathrm{p} 38$ MAPK signaling pathway might play an essential role in DPSC-Exos induced angiogenesis.

Interestingly, the proteomic data indicated that expression of FGD5, a pro-angiogenesis protein, were 2.299 times higher in DPSC-Exos than in DPSCs (Additional file 2). FGD5 is known to mediate proangiogenic action of vascular endothelial growth factor (VEGF) in human vascular endothelial cells by inducing the activation of $\mathrm{Cdc} 42^{24}$. Western blotting analysis also veirified that FGD5 was enriched in DPSC-Exos (Figure 4G), which may be a potential candidate mediating pro-angiogenic function of DPSCExos.

\section{Inhibition of the p38 MAPK signaling pathway decreased DPSC-Exos-induced angiogenesis}

To examine whether p38 MAPK signaling pathway was involved in DPSC-Exos induced angiogenesis, we treated HUVECs with SB203580 to inhibit p38 MAPK pathway before stimulation by DPSC-Exos. Hsp27 is

a terminal substrate of the $\mathrm{p} 38$ MAPK pathway ${ }^{25,26}$, which is related to positive regulation of angiogenesis as well as positive regulation of blood vessel endothelial cell migration ${ }^{27}$. Once we inhibited the p38 MAPK signaling pathways by SB203580, protein levels of hsp27 and phosphorylated-hsp27 were significantly reduced in HUVECs (Figure 5A, B). Transwell migration assay (Figure 5C, D) and scratch wound assay (Figure 5E, F) showed a decreased capacity of DPSC-Exos to promote cell migration when HUVECs was pre-treated with SB203580. From CCK-8 assay, proliferation of HUVECs improved by DPSCExos also shrunk (Figure 5G). In tube formation assay, the enhanced tube formation ability of HUVEC stimulated by DPSC-Exos was reduced by SB203580 (Figure 5H, I, J). These outcomes all above confirmed that p38 MAPK signaling pathway was involved in DPSC-Exos-induced angiogenesis.

\section{Discussion}

In this work, we found for the first time that DPSC-Exos could effectively augment the functional properties of HUVECs and accelerate cutaneous wound healing in mice. In the process of angiogenesis induced by DPSC-Exos, Cdc42/p38 MAPK pathway played a crucial role, as the pro-angiogenic effects of DPSC-Exos could be attenuated by inhibition of p38 MAPK pathway (Figure 6). 
We found that DPSC-Exos can accelerate cutaneous wound healing by enhancing the development of new blood vessels in the wound site. As reported, DPSC-derived exosomes showed several potentials in bone regeneration, neuroprotection and anti-inflammation ${ }^{28-30}$. In our previous study, DPSC-Exos were found to promote odontogenic differentiation via TGF $\beta 1 /$ smads signaling pathway by downregulating LTBP $1^{20}$. The present study showed that injection of DPSC-Exos triggered angiogenesis by evaluating the vascular marker CD31 in the wound site. Angiogenesis at the wound site is a critical determinant of wound healing processes, since neovascularization ensures oxygen and nutrition delivery, thus establishing a favorable environment for wound healing. The stimulation of new blood vessel development is a helpful therapeutic target for tissue regeneration.

Recently, exosomes from hypoxia-preconditioned human adipose MSCs have been found to carry over 30 kinds of angiogenesis-related proteins and improve neovascularization around the graft tissue ${ }^{31}$. Exosomes from MCSs derived from human umbilical cord, expressing a2M, TLN1, ANK1 and other proteins, involved in damage repairing ${ }^{32}$. In our study, proteomic analysis showed that 1619 differentially expressed proteins were detected in DPSC-Exo. This meant that there may be potential functional proteins among them, which involved in different processes of wound healing, and should be worthy of further exploration.

GO term enrichment analysis showed that DPSC-Exos were enriched in the proteins that are involved in regulation of wound healing-related biological processes, such as positive regulation of cell motility, migration, proliferation, vasculature development and angiogenesis. Additionally, we conducted several in vitro experiments to demonstrate that DPSC-Exos effectively enhanced the migration, proliferation and tube formation ability of HUVECs. New blood vessel formation, which involves in endothelial cell proliferation, migration, and branching to form capillaries, requires a dynamic regulated interaction between endothelial cells, angiogenesis factors, and the surrounding extracellular matrix ${ }^{33}$. It's known that some exosomes can regulate the angiogenic function of recipient endothelial cells by transferring exosomal proteins, RNAs and microRNAs into cytoplasm of them ${ }^{34}$. Such exosomes represent a highly attractive delivery vehicle for any proteins or RNAs through which we wish to exert their therapeutic effects.

Previous studies have demonstrated that MSC-Exos could facilitate wound repair, but some of the underlying mechanisms remain unclear ${ }^{32,35,36}$. In the present study, we focused on identifying the underlying mechanism by which infusion of DPSC-Exos initiates endothelial cell-mediated wound healing. KEGG pathway analysis found that exosomal proteins were enriched in several pathways, including MAPK pathway. Our western blotting confirmed that DPSC-Exos could induce significant increases in the protein level of Cdc42 and phosphorylation of p38 in HUVECs. Cdc42 is a Rho-family GTPase regulating actin dynamics and cell proliferation ${ }^{37}$. Moreover, it is an important activator of p38 MAPK pathway ${ }^{38}$. Sequential activation of Cdc42/p38 MAPK signaling pathway was essential to VEGFinduced actin reorganization in HUVECs ${ }^{39,40}$. Several studies have indicated that p38 MAPK activation mediated angiogenesis of endothelial cells by modulating cell migration and proliferation ${ }^{25,41}$. Thus, we 
hypothesized that DPSC-Exos increased the angiogenic activities of HUVECs through p38 MAPK pathway. We found that stimulation of DPSC-Exos could increase the angiogenic activities of HUVECs, whereas these effects were attenuated with the inhibition of p38 MAPK signaling pathway. Taken together, our findings demonstrated that DPSC-Exos increased the migration, proliferation, and capillary formation capacity of endothelial cells via Cdc42/p38 MAPK signaling pathway, thereby enhancing cutaneous wound healing in mice.

It has been reported that exosomal proteins could activate p38 MAPK pathway and promotes angiogenesis ${ }^{42}$. Moreover, AKT/mTOR pathway, JAK2/STAT3 pathway and PKA signaling pathway were associated with MSC-exos-induced angiogenesis ${ }^{43-45}$. Our data confirmed that the positive effects of DPSC-Exos on HUVECs that SB203580 were not entirely abolished by inhibition of p38 MAPK signaling pathway, which suggested that other pathways were involved in the regulation of neovascularization.

Furthermore, we sought to determine the key component that participated in the modulation of DPSC-Exostimulated angiogenic activities of HUVECs. We noticed that the expression of FGD5, a pro-angiogenic protein, was markedly higher in DPSC-Exos. FGD5, known to be conducive to pro-angiogenesis processes in endothelial cells, is a Rho guanine-nucleotide exchange factor (Rho GEF) which catalyzes the exchange of GDP for GTP and leads to the activation of its target Rho protein $\mathrm{Cdc} 42^{46}$. Previous study demonstrated that FGD5 regulates Cdc42 activity and played a key role on the mediation of proangiogenic action of $\mathrm{VEGF}^{24}$. FGD5-mediated activation of Cdc42 in endothelial cells could protect VEGFR2 from degradation and regulates cytoskeletal dynamics ${ }^{47}$. To investigate the molecular mechanisms of FGD5 from DPSC-Exos on the angiogenesis of HUVECs, future studies will be necessary.

\section{Conclusions}

In this study, we demonstrated that DPSC-Exos promoted the angiogenic properties of endothelial cells via Cdc42/p38 MAPK signaling pathway, thereby enhancing cutaneous wound healing in mice. DPSC-Exo based therapy could possibly represent a useful tool in the field of soft tissue regeneration. However, more studies need to be developed before DPSC-Exo can be put into clinic.

\section{Abbreviations}

DPSCs: Dental pulp stem cells

HUVECs: Human umbilical vein endothelial cells

MSCs: Mesenchymal stem cells

Cdc42: cell division cycle 42

FGD 5: FYVE, RhoGEF and PH Domain Containing 5 
VEGF: Vascular endothelial growth factor

TMT: Tandem Mass Tags

OD: Optical density

NTA: Nanoparticle tracking analysis

TEM: Transmission electron microscopy

\section{Declarations}

\section{Acknowledgements}

Not applicable.

\section{Author contributions}

$\mathrm{XH}$ and $\mathrm{JZ}$ designed the study. ZZ and JZ performed the experiments and collected the data. DL and YC helped with the experiments. $\mathrm{ZZ}$ analyzed the data and prepared the manuscript. $\mathrm{XH}$ and $\mathrm{JZ}$ revised the manuscript.

\section{Funding}

This work was supported by the National Natural Science Foundation of China (Grant No.11772361 and 81700950), and Guangdong Basic and Applied Basic Research Foundation (Grant No.2019A1515011289).

\section{Availability of data and materials}

The data are available from the corresponding author upon request.

\section{Ethic approval and consent to participate}

All animal procedures were approved by the Ethics Committee of Sun Yat-sen University.

\section{Consent for publication}

Not applicable. 


\section{Competing Interests}

The authors deny any conflicts of interest related to this study.

\section{References}

1. Strecker-McGraw MK, Jones TR, Baer DG. Soft tissue wounds and principles of healing. Emerg Med Clin North Am Feb. 2007;25(1):1-22. doi:10.1016/j.emc.2006.12.002.

2. Rodrigues M, Kosaric N, Bonham CA, Gurtner GC. Wound Healing: A Cellular Perspective. Physiol Rev Jan. 2019;1(1):665-706. doi:10.1152/physrev.00067.2017. 99 ) .

3. Gurtner GC, Werner S, Barrandon Y, Longaker MT. Wound repair and regeneration. Nature May. 2008;15(7193):314-21. doi:10.1038/nature07039. 453 ) .

4. Li J, Zhang YP, Kirsner RS. Angiogenesis in wound repair: angiogenic growth factors and the extracellular matrix. Microsc Res Tech Jan. 2003;1(1):107-14. doi:10.1002/jemt.10249. 60 ) .

5. Ding J, Wang X, Chen B, Zhang J, Xu J. Exosomes Derived from Human Bone Marrow Mesenchymal Stem Cells Stimulated by Deferoxamine Accelerate Cutaneous Wound Healing by Promoting Angiogenesis. Biomed Res Int. 2019;2019:9742765. doi:10.1155/2019/9742765.

6. Li X, Xie X, Lian W, et al. Exosomes from adipose-derived stem cells overexpressing Nrf2 accelerate cutaneous wound healing by promoting vascularization in a diabetic foot ulcer rat model. Exp Mol Med Apr. 2018;13(4):1-14. doi:10.1038/s12276-018-0058-5. 50 ) .

7. Hu Y, Rao SS, Wang ZX, et al. Exosomes from human umbilical cord blood accelerate cutaneous wound healing through miR-21-3p-mediated promotion of angiogenesis and fibroblast function. Theranostics. 2018;8(1):169-84. doi:10.7150/thno.21234.

8. Théry C, Zitvogel L, Amigorena S. Exosomes: composition, biogenesis and function. Nat Rev Immunol Aug. 2002;2(8):569-79. doi:10.1038/nri855.

9. Yang B, Chen Y, Shi J. Exosome Biochemistry and Advanced Nanotechnology for Next-Generation Theranostic Platforms. Adv Mater Jan. 2019;31(2):e1802896. doi:10.1002/adma.201802896.

10. Gonzalez-King H, García NA, Ontoria-Oviedo I, Ciria M, Montero JA, Sepúlveda P. Hypoxia Inducible Factor-1a Potentiates Jagged 1-Mediated Angiogenesis by Mesenchymal Stem Cell-Derived Exosomes. Stem Cells Jul. 2017;35(7):1747-59. doi:10.1002/stem.2618.

11. Liu J, Yan Z, Yang F, et al. Exosomes Derived from Human Umbilical Cord Mesenchymal Stem Cells Accelerate Cutaneous Wound Healing by Enhancing Angiogenesis through Delivering Angiopoietin-2. Stem Cell Rev Rep Apr. 2021;17(2):305-17. doi:10.1007/s12015-020-09992-7.

12. Chen $\mathrm{CY}$, Rao SS, Ren $\mathrm{L}$, et al. Exosomal DMBT1 from human urine-derived stem cells facilitates diabetic wound repair by promoting angiogenesis. Theranostics. 2018;8(6):1607-23. doi:10.7150/thno.22958.

13. Tsutsui TW. Dental Pulp Stem Cells: Advances to Applications. Stem Cells Cloning. 2020;13:33-42. doi:10.2147/sccaa.S166759. 
14. Botelho J, Cavacas MA, Machado V, Mendes JJ. Dental stem cells: recent progresses in tissue engineering and regenerative medicine. Ann Med Dec. 2017;49(8):644-51. doi:10.1080/07853890.2017.1347705.

15. Martínez-Sarrà E, Montori S, Gil-Recio C, et al. Human dental pulp pluripotent-like stem cells promote wound healing and muscle regeneration. Stem Cell Res Ther Jul. 2017;27(1):175. doi:10.1186/s13287-017-0621-3. 8 ) .

16. Mead B, Logan A, Berry M, Leadbeater W, Scheven BA. Concise Review: Dental Pulp Stem Cells: A Novel Cell Therapy for Retinal and Central Nervous System Repair. Stem Cells Jan. 2017;35(1):61-7. doi:10.1002/stem.2398.

17. Huang CC, Narayanan R, Alapati S, Ravindran S. Exosomes as biomimetic tools for stem cell differentiation: Applications in dental pulp tissue regeneration. Biomaterials Dec. 2016;111:103-15. doi:10.1016/j.biomaterials.2016.09.029.

18. Jarmalavičiūtè A, Tunaitis V, Pivoraitè U, Venalis A, Pivoriūnas A. Exosomes from dental pulp stem cells rescue human dopaminergic neurons from 6-hydroxy-dopamine-induced apoptosis. Cytotherapy Jul. 2015;17(7):932-9. doi:10.1016/j.jcyt.2014.07.013.

19. Swanson WB, Zhang Z, Xiu K, et al. Scaffolds with controlled release of pro-mineralization exosomes to promote craniofacial bone healing without cell transplantation. Acta BiomaterDec. 2020;118:215-32. doi:10.1016/j.actbio.2020.09.052.

20. Hu X, Zhong Y, Kong Y, Chen Y, Feng J, Zheng J. Lineage-specific exosomes promote the odontogenic differentiation of human dental pulp stem cells (DPSCs) through TGF $\beta 1 /$ smads signaling pathway via transfer of microRNAs. Stem Cell Res Ther Jun. 2019;13(1):170. doi:10.1186/s13287-019-1278-x. $10)$.

21. Kumar S, Jiang MS, Adams JL, Lee JC. Pyridinylimidazole compound SB 203580 inhibits the activity but not the activation of p38 mitogen-activated protein kinase. Biochem Biophys Res Commun Oct. 1999;5(3):825-31. doi:10.1006/bbrc.1999.1454. 263 ) .

22. Liu Z, Wu H, Jiang K, et al. MAPK-Mediated YAP Activation Controls Mechanical-Tension-Induced Pulmonary Alveolar Regeneration. Cell Rep Aug. 2016;16(7):1810-9. doi:10.1016/j.celrep.2016.07.020. 16 ) .

23. Koch S, Claesson-Welsh L. Signal transduction by vascular endothelial growth factor receptors. Cold Spring Harb Perspect Med Jul. 2012;2(7):a006502. doi:10.1101/cshperspect.a006502.

24. Kurogane $Y$, Miyata M, Kubo Y, et al. FGD5 mediates proangiogenic action of vascular endothelial growth factor in human vascular endothelial cells. Arterioscler Thromb Vasc Biol Apr. 2012;32(4):988-96. doi:10.1161/atvbaha.111.244004.

25. Rousseau S, Houle F, Landry J, Huot J. p38 MAP kinase activation by vascular endothelial growth factor mediates actin reorganization and cell migration in human endothelial cells. Oncogene Oct. 1997;15(18):2169-77. doi:10.1038/sj.onc.1201380.

26. Armstrong SC, Delacey M, Ganote CE. Phosphorylation state of hsp27 and p38 MAPK during preconditioning and protein phosphatase inhibitor protection of rabbit cardiomyocytes. J Mol Cell 
Cardio/ Mar. 1999;31(3):555-67. doi:10.1006/jmcc.1998.0891.

27. Evans IM, Britton G, Zachary IC. Vascular endothelial growth factor induces heat shock protein (HSP) 27 serine 82 phosphorylation and endothelial tubulogenesis via protein kinase $D$ and independent of p38 kinase. Cell Signal Jul. 2008;20(7):1375-84. doi:10.1016/j.cellsig.2008.03.002.

28. Xie L, Guan Z, Zhang M, et al. Exosomal circLPAR1 Promoted Osteogenic Differentiation of Homotypic Dental Pulp Stem Cells by Competitively Binding to hsa-miR-31. Biomed Res Int. 2020;2020:6319395. doi:10.1155/2020/6319395.

29. Venugopal C, Rai KS, Pinnelli KS, Kutty VB, Dhanushkodi BM. A. Neuroprotection by Human Dental Pulp Mesenchymal Stem Cells: From Billions to Nano. Curr Gene Ther. 2018;18(5):307-23. doi:10.2174/1566523218666180913152615.

30. Pivoraitè U, Jarmalavičiūtè A, Tunaitis V, et al. Exosomes from Human Dental Pulp Stem Cells Suppress Carrageenan-Induced Acute Inflammation in Mice. Inflammation Oct. 2015;38(5):1933-41. doi:10.1007/s10753-015-0173-6.

31. Han Y, Ren J, Bai Y, Pei X, Han Y. Exosomes from hypoxia-treated human adipose-derived mesenchymal stem cells enhance angiogenesis through VEGF/VEGF-R. Int J Biochem Cell Biol Apr. 2019;109:59-68. doi:10.1016/j.biocel.2019.01.017.

32. Bakhtyar N, Jeschke MG, Herer E, Sheikholeslam M, Amini-Nik S. Exosomes from acellular Wharton's jelly of the human umbilical cord promotes skin wound healing. Stem Cell Res Ther Jul. 2018;13(1):193. doi:10.1186/s13287-018-0921-2. 9 ) .

33. Tonnesen MG, Feng X, Clark RA. Angiogenesis in wound healing. J Investig Dermatol Symp Proc. Dec 2000;5(1):40-6. doi:10.1046/j.1087-0024.2000.00014.x.

34. Ribeiro MF, Zhu H, Millard RW, Fan GC. Exosomes Function in Pro- and Anti-Angiogenesis. Curr Angiogenes. 2013;2(1):54-9. doi:10.2174/22115528113020020001.

35. Wu P, Zhang B, Shi H, Qian H, Xu W. MSC-exosome: A novel cell-free therapy for cutaneous regeneration. Cytotherapy Mar. 2018;20(3):291-301. doi:10.1016/j.jcyt.2017.11.002.

36. Yu M, Liu W, Li J, et al. Exosomes derived from atorvastatin-pretreated MSC accelerate diabetic wound repair by enhancing angiogenesis via AKT/eNOS pathway. Stem Cell Res Ther Aug. 2020;12(1):350. doi:10.1186/s13287-020-01824-2. 11 ) .

37. Liu S, Uppal H, Demaria M, Desprez PY, Campisi J, Kapahi P. Simvastatin suppresses breast cancer cell proliferation induced by senescent cells. Sci Rep Dec. 2015;14:5:17895. doi:10.1038/srep17895.

38. Liu J, He X, Corbett SA, et al. Integrins are required for the differentiation of visceral endoderm. J Cell Sci Jan. 2009;15(Pt 2):233-42. doi:10.1242/jcs.037663. 122 ) .

39. Lamalice L, Houle F, Jourdan G, Huot J. Phosphorylation of tyrosine 1214 on VEGFR2 is required for VEGF-induced activation of Cdc42 upstream of SAPK2/p38. Oncogene Jan. 2004;15(2):434-45. doi:10.1038/sj.onc.1207034. 23 ) .

40. Lamalice L, Houle F, Huot J. Phosphorylation of Tyr1214 within VEGFR-2 triggers the recruitment of Nck and activation of Fyn leading to SAPK2/p38 activation and endothelial cell migration in response to VEGF. J Biol Chem Nov. 2006;10(45):34009-20. doi:10.1074/jbc.M603928200. 281 ) . 
41. Feng PC, Ke XF, Kuang HL, Pan LL, Ye Q, Wu JB. BMP2 secretion from hepatocellular carcinoma cell HepG2 enhances angiogenesis and tumor growth in endothelial cells via activation of the MAPK/p38 signaling pathway. Stem Cell Res Ther Aug. 2019;6(1):237. doi:10.1186/s13287-019-1301-2. 10 ) .

42. Maji S, Chaudhary P, Akopova I, et al. Exosomal Annexin II Promotes Angiogenesis and Breast Cancer Metastasis. Mol Cancer Res Jan. 2017;15(1):93-105. doi:10.1158/1541-7786.Mcr-16-0163.

43. Liang B, Liang JM, Ding JN, Xu J, Xu JG, Chai YM. Dimethyloxaloylglycine-stimulated human bone marrow mesenchymal stem cell-derived exosomes enhance bone regeneration through angiogenesis by targeting the AKT/mTOR pathway. Stem Cell Res Ther Nov. 2019;20(1):335. doi:10.1186/s13287019-1410-y. 10 ) .

44. Zhou X, Yan T, Huang $C$, et al. Melanoma cell-secreted exosomal miR-155-5p induce proangiogenic switch of cancer-associated fibroblasts via SOCS1/JAK2/STAT3 signaling pathway. J Exp Clin Cancer Res Oct. 2018;3(1):242. doi:10.1186/s13046-018-0911-3. 37 ) .

45. Xue C, Shen Y, Li X, et al. Exosomes Derived from Hypoxia-Treated Human Adipose Mesenchymal Stem Cells Enhance Angiogenesis Through the PKA Signaling Pathway. Stem Cells Dev Apr. 2018;1(7):456-65. doi:10.1089/scd.2017.0296. 27 ) .

46. Park S, Guo Y, Negre J, et al. Fgd5 is a Rac1-specific Rho GEF that is selectively inhibited by aurintricarboxylic acid. Small GTPases Mar. 2021;12(2):147-60. doi:10.1080/21541248.2019.1674765.

47. Heldin J, O'Callaghan P, Hernández Vera R, Fuchs PF, Gerwins P, Kreuger J. FGD5 sustains vascular endothelial growth factor A (VEGFA) signaling through inhibition of proteasome-mediated VEGF receptor 2 degradation. Cell Signa/ Dec. 2017;40:125-32. doi:10.1016/j.cellsig.2017.09.009.

\section{Figures}

A

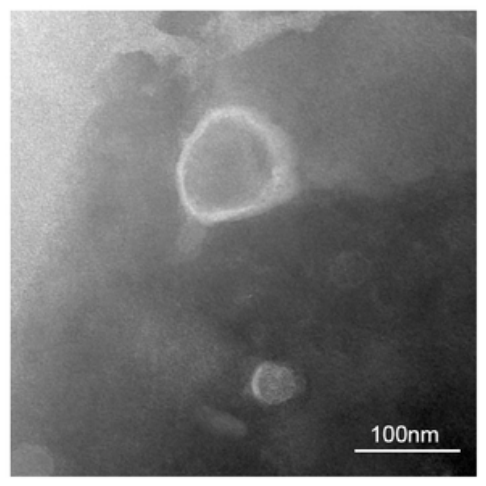

B

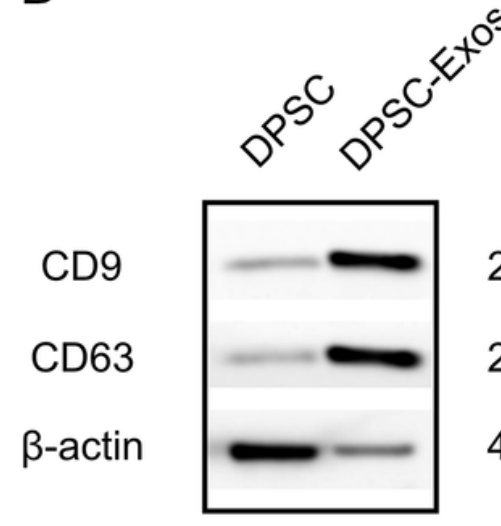

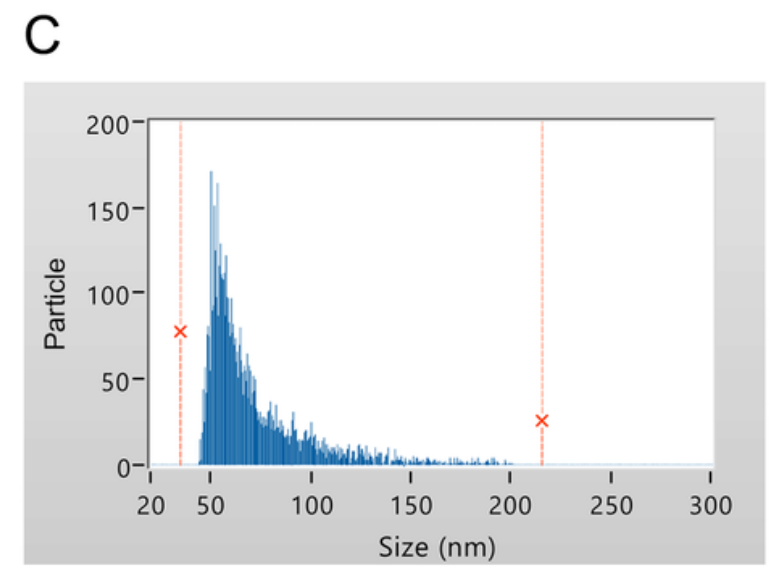

\section{Figure 1}

Identification of DPSC-Exos. (A) The morphology of DPSC-Exos was observed by TEM. Scale bar: 100nm. (B) Western blot analysis of exosomal markers (CD9, CD63) and cytosolic marker ( $\beta$-actin) expression 
from DPSC-Exos. (C) Nanoparticle size distribution of DPSC-Exos detected by NTA.

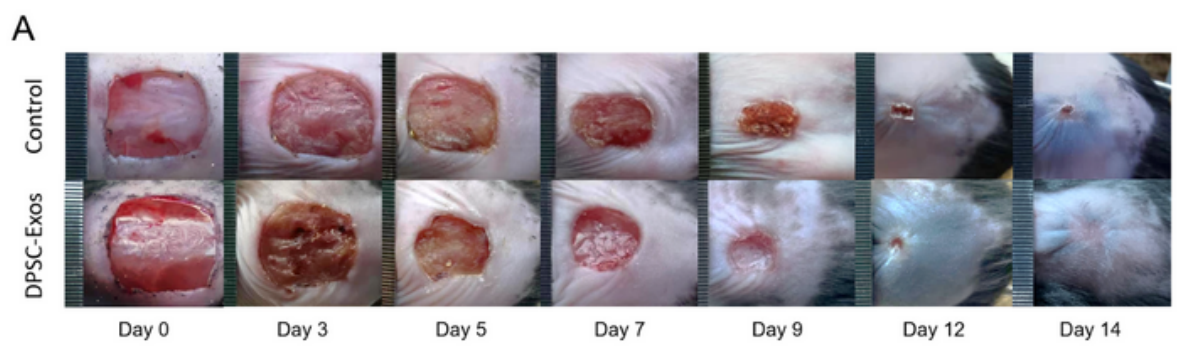

B

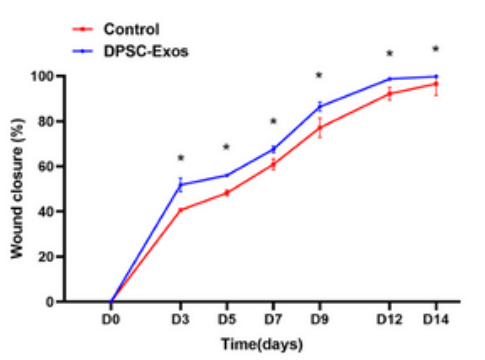

C

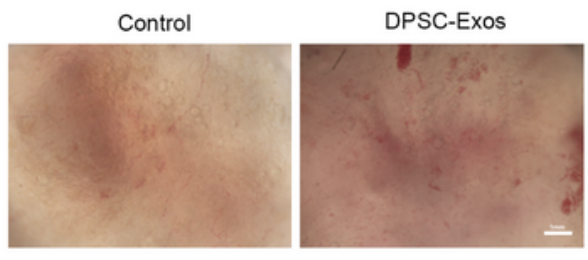

D
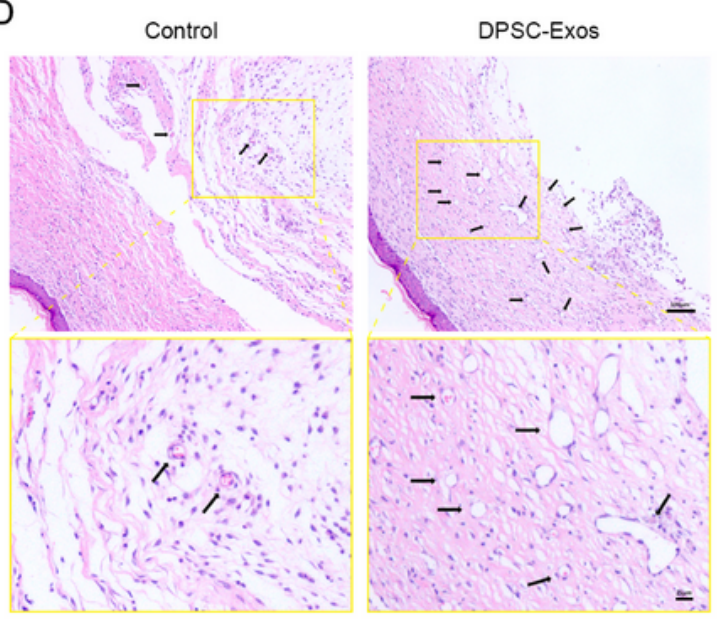

E

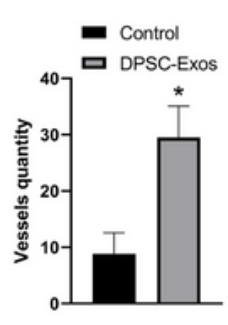

$\mathrm{F}$
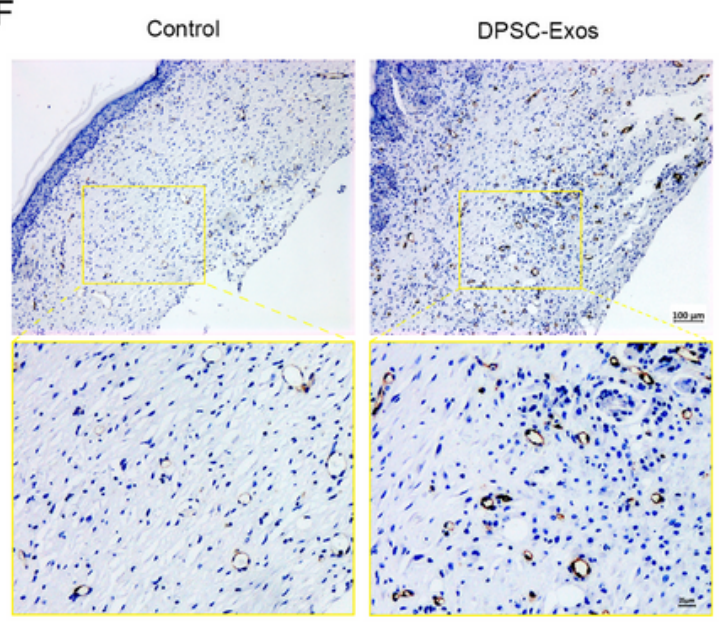

G

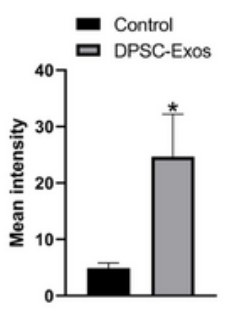

Figure 2

DPSC-Exos accelerated cutaneous wound healing in mice by promoting angiogenesis. (A) Gross view and quantification of wounds area treated with PBS and DPSC-Exos at days 3, 5, 7, 9, 12, 14 post-wounding. Scale bar: $1 \mathrm{~cm}$. (B) The rate of wound-closure in wounds receiving DPSC-Exos treatments was significantly higher at the indicated times $(n=3)$. (C) Gross view of wounds treated with PBS and DPSC- 
Exos at day 14 post-wounding from the undersurface. More newly formed blood vessels were detected in the wound sites of DPSC-Exos-treated group. Scale bar: $1 \mathrm{~mm}$. (D, E) H\&E staining of wound sections treated with PBS and DPSC-Exos at 14 days after operation. The black arrows indicated newly formed blood vessels. The vessels quantity in DPSC-Exos-treated group was larger than control group $(n=3)$. Scale bar: $100 \mu \mathrm{m}$. $(F, G)$ Immunohistochemical staining for CD31 in wound sections treated with PBS and DPSC-Exos at 14 days after operation. Higher expression of CD31 was shown in DPSC-Exos-treated group $(n=3)$. Scale bar: $100 \mu m$. ${ }^{*}<<0.05$.

A

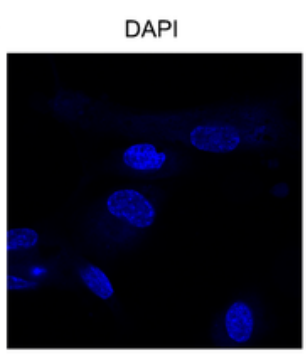

PKH26

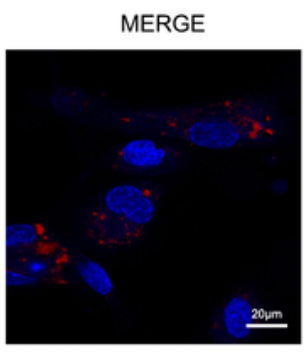

B

Control

DPSC-Exos

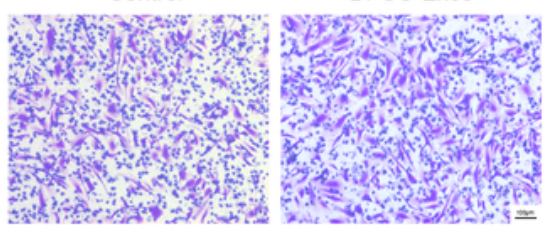

D

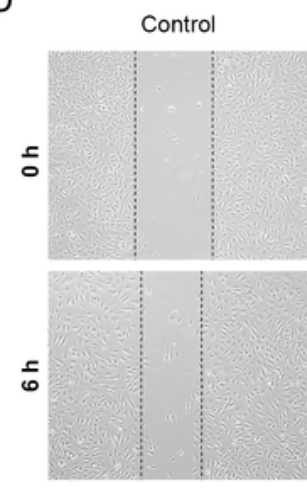

DPSC-Exos

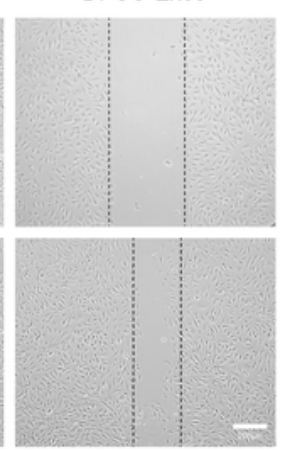

C

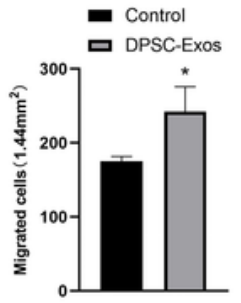

E

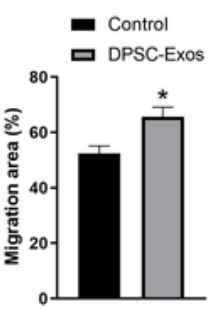

F

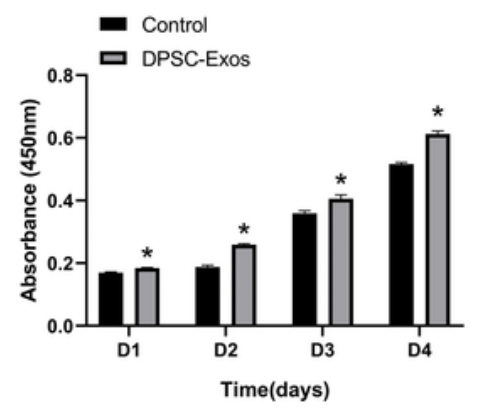

G

H

Control

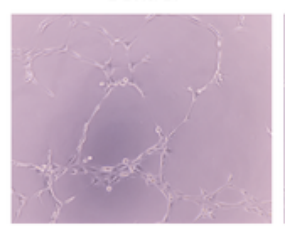

DPSC-Exos

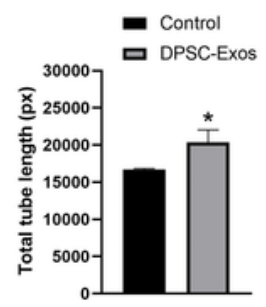

I

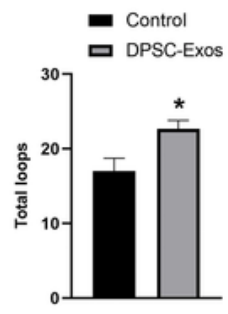




\section{Figure 3}

DPSC-Exos enhanced the angiogenic activities of HUVECs. (A) Endocytosis of exosomes by HUVECs was visualized by fluorescent dyeing with PKH26. (B, C) The migration of HUVECs stimulated by DPSC-Exos was increased $(n=3)$. Scale bar: $100 \mu m$. (D, E) Representative images of scratch wound assay in HUVECs treated with DPSC-Exos or PBS. The remain area of DPSC-Exo-treated group was smaller than control group $(n=3)$. Scale bar: $100 \mu \mathrm{m}$. (F) The proliferation of HUVECs receiving different treatments were tested by CCK-8 analysis. DPSC-Exo-treated group showed a greater proliferative capacity than control group $(n=3) .(G, H, I)$ Representative images of the tube formation assay on Matrigel in HUVECs treated with DPSC-Exos or PBS. The total tube length and quantity of total loops in DPSC-Exo-treated group were larger than control group $(n=3)$. Scale bar: $100 \mu m$. ${ }^{*}<<0.05$. 
A

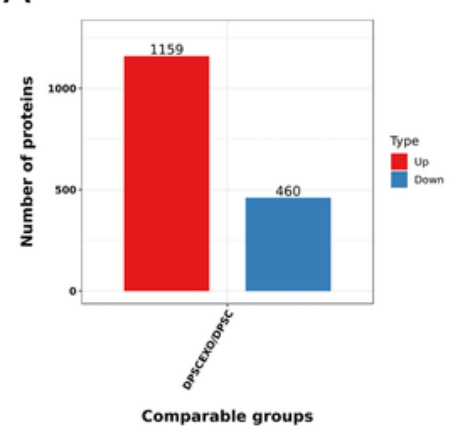

B

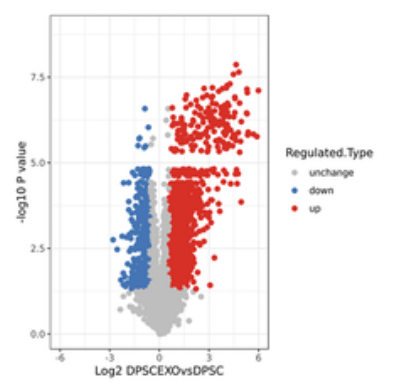

C

\section{Biological Process}

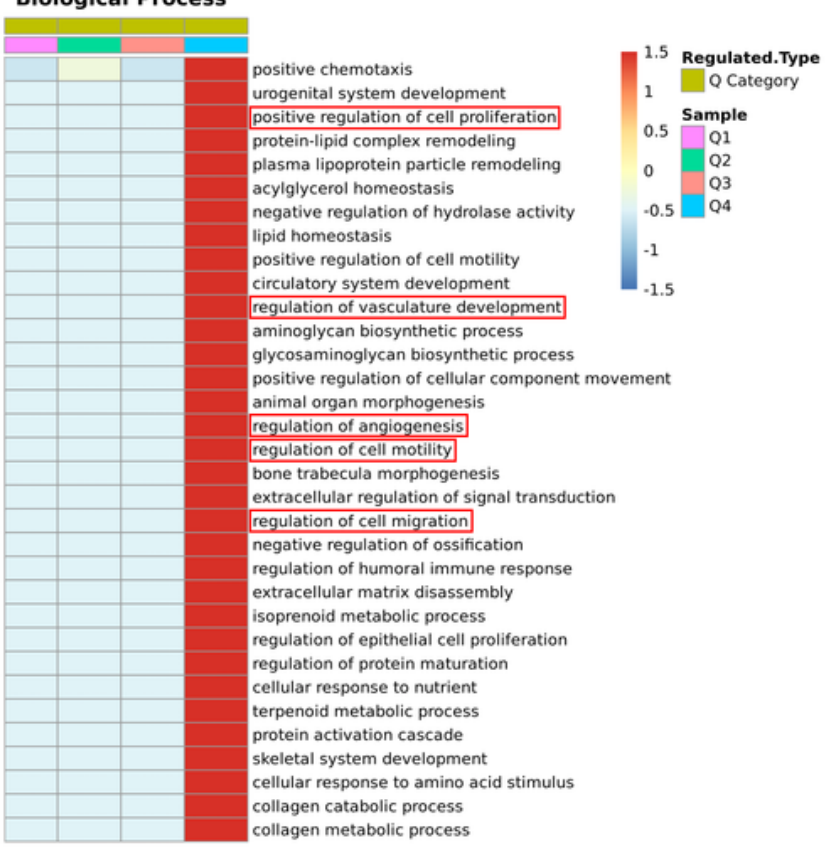

D

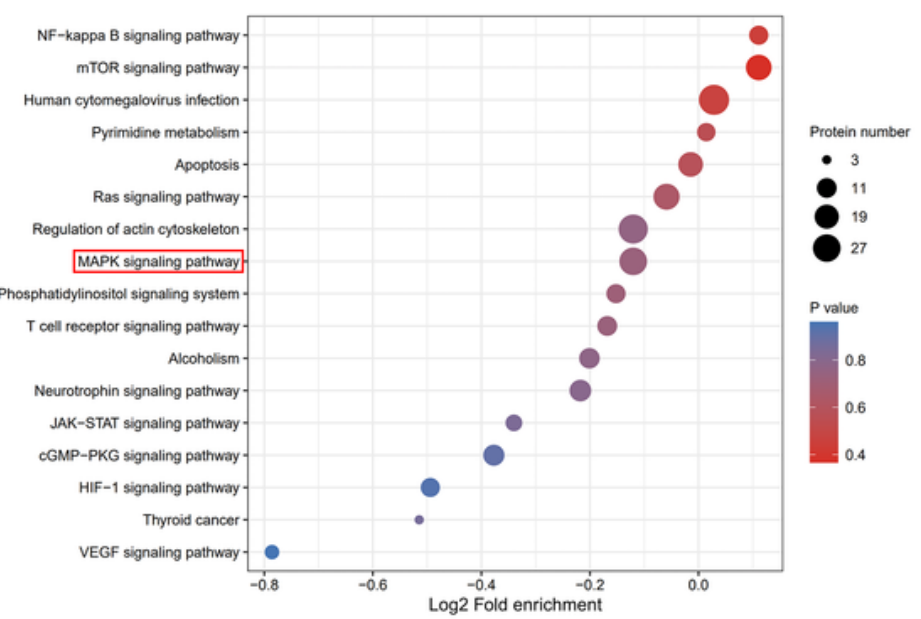

$\mathrm{E}$

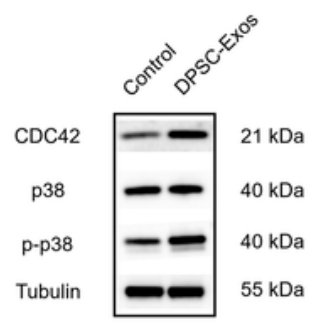

$\mathrm{F}$
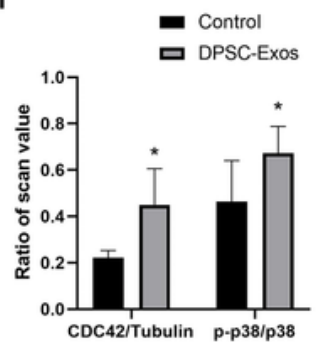

G

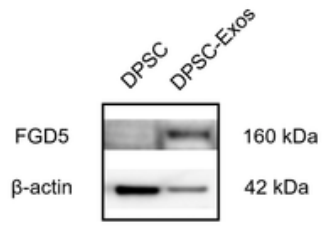

\section{Figure 4}

Expression and function of the proteins identified by TMT-labeled quantitative proteomic in DPSC-Exos. (A) The number of differentially expressed proteins in DPSC-Exos compared with DPSCs identified by TMT-labeled quantitative proteomic. (B) The volcano map of differentially expressed proteins. (C) Differentially expressed proteins were categorized according to the biological process (GO term) they were involved in, several of them were related to wound healing. (D) KEGG pathway analysis of DPSC- 
Exos showed that enriched proteins involved in multiple signal transductions, including MAPK signaling pathway. (E, F) The expression of Cdc42, p38 and phosphorylated-p38 (p-p38) in HUVECs was higher when treated with DPSC-Exos $(n=3) .{ }^{*}<0.05$. (G) Western blot analysis of pro-angiogenic protein (FGD5) and cytosolic marker ( $\beta$-actin) expression from DPSC and DPSC-Exos.

A

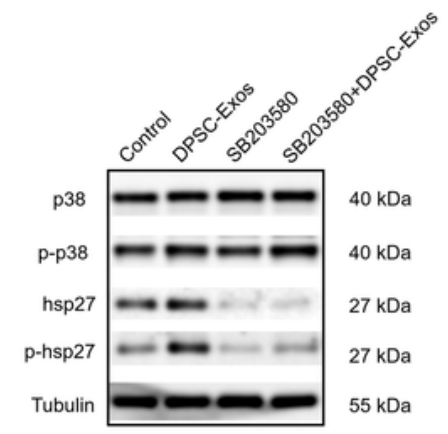

C

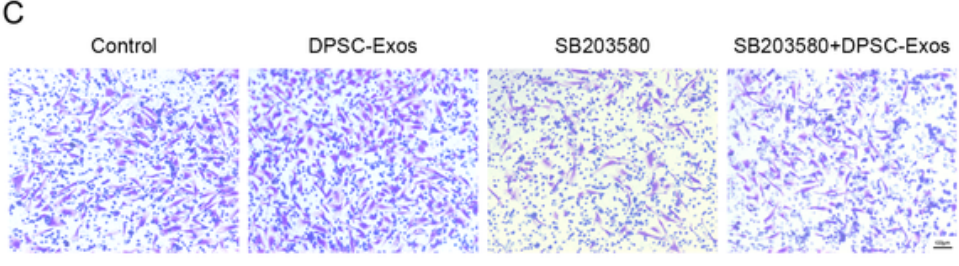

E
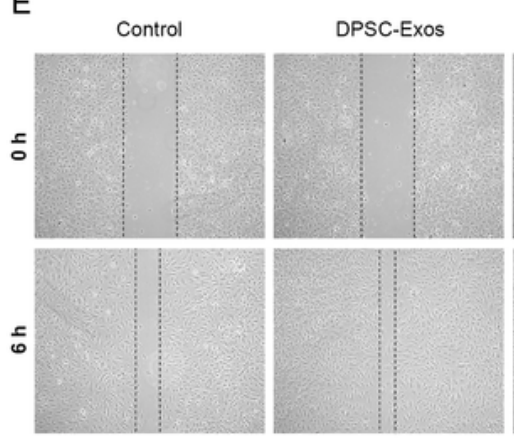

G

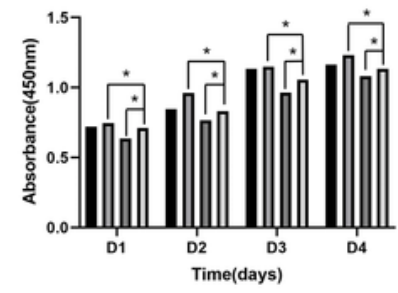

$\mathrm{H}$

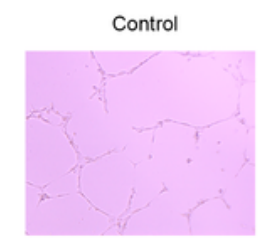

DPSC-Exos
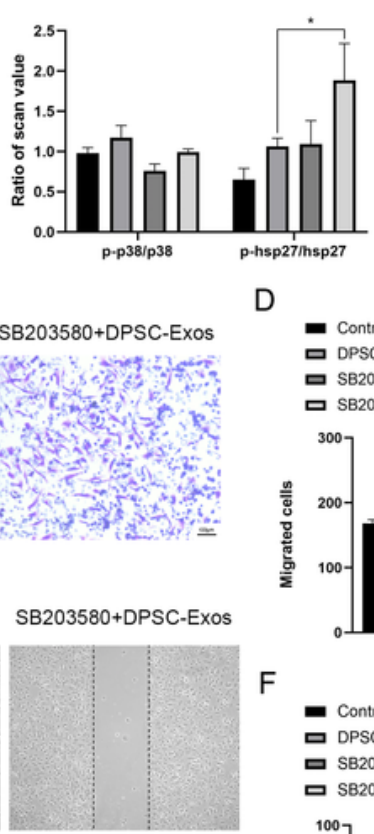

D

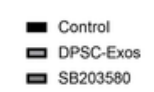

ㅁ SB203580+DPSC-Exos

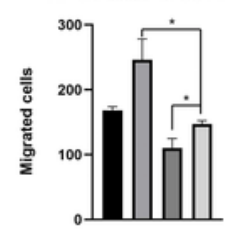

$\mathrm{F}$

- Control

口 DPSC-Exos

口 $\mathrm{SB} 203580$

口 SB203580+DPSC-Exos

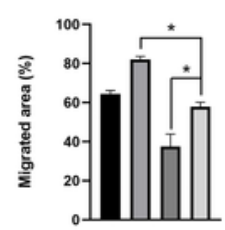

I

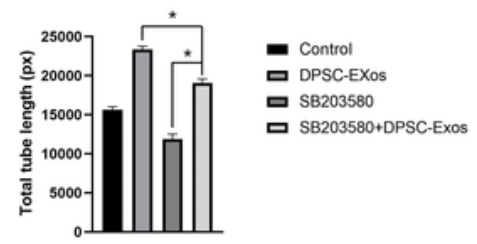

SB203580

SB203580+DPSC-Exos
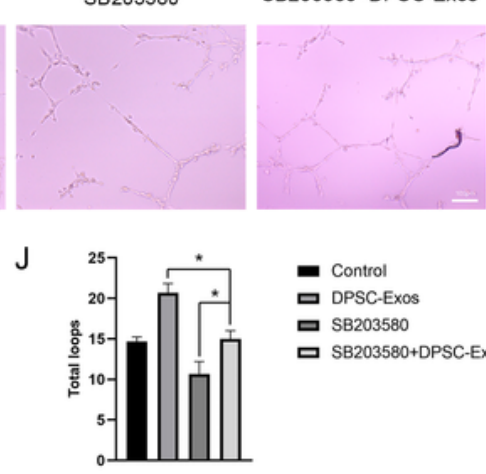

- Control

ㅁ DPSC-Exos

- SB203580

口 SB203580+DPSC-Exos

Figure 5 
DPSC-Exos induced angiogenesis activation through p38 MAPK to promote wound healing. (A, B) HUVECs were treated with DPSC-Exos in the presence or absence of SB203580. The expression of p38, phosphorylated-p38 (p-p38), hsp27 and phosphorylated-hsp27 (p-hsp27) was detected by Western blot $(n=3)$. (C, D) The migration of HUVECs stimulated by DPSC-Exos or an equal volume of PBS in the presence or absence of SB203580 $(n=3)$. Scale bar: 100 $\mu$ m. $(E, F)$ Representative images of scratch wound assay in HUVECs treated with DPSC-Exos or PBS in the presence or absence of SB203580 $(n=3)$. Scale bar: $100 \mu \mathrm{m}$. (G) The proliferation of HUVECs receiving different treatments were tested by CCK-8 analysis $(n=3)$. $(H, I, J)$ Representative images of the tube formation assay on Matrigel in HUVECs treated with DPSC-Exos or PBS $(n=3)$. Scale bar: $100 \mu m$. ${ }^{*}<<0.05$.

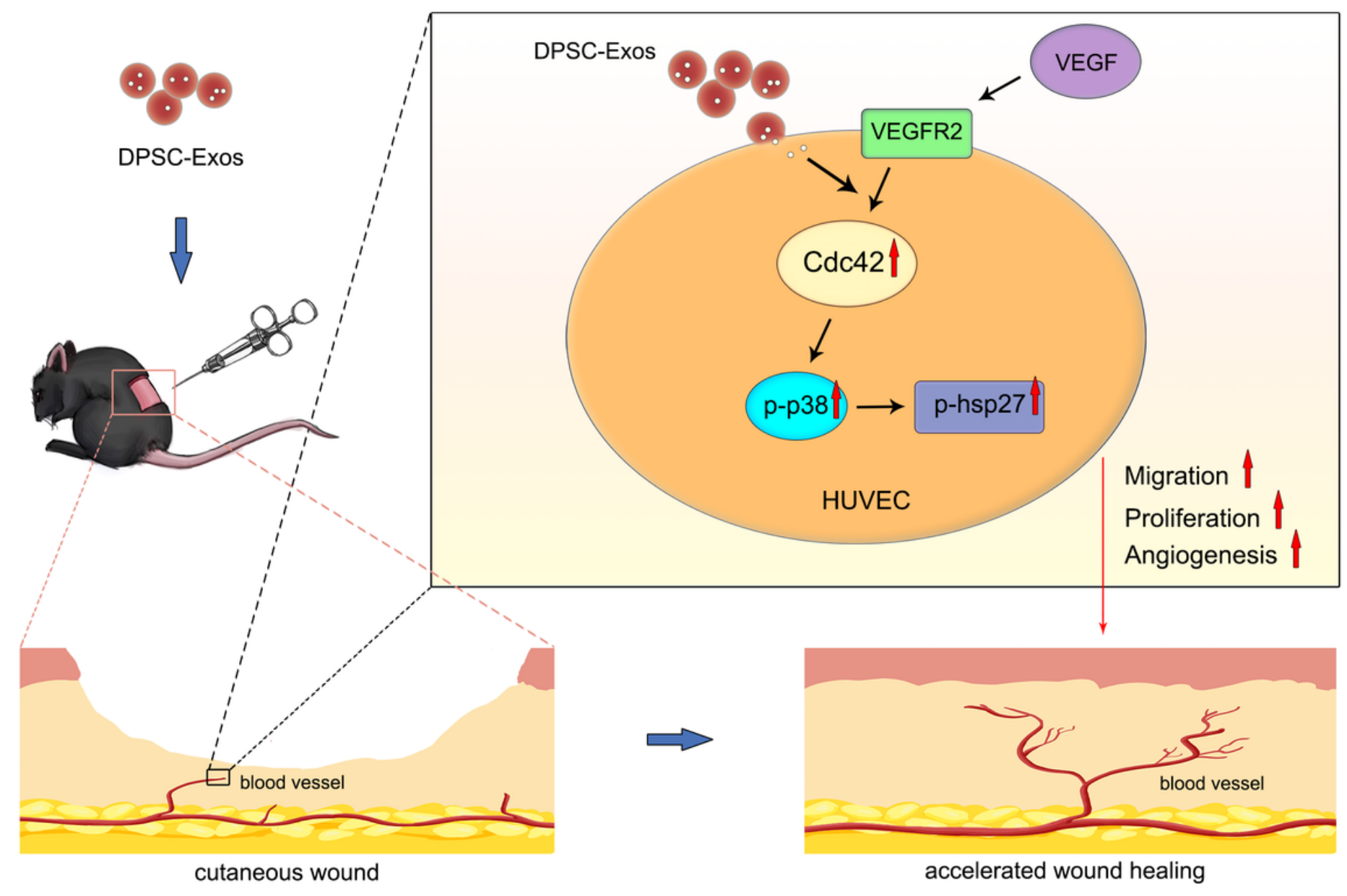

Figure 6

Schematic model of the function of DPSC-Exos in accelerating cutaneous wound healing in mice by inducing angiogenesis of HUVECs via Cdc42/p38 MAPK signaling pathway.

\section{Supplementary Files}

This is a list of supplementary files associated with this preprint. Click to download.

- Differentiallyexpressedstatistics.xlsx 
- MAPKpathwayrelatedproteinAnnotationcombine.xIsx 